\title{
Three-IncRNA signature is a potential prognostic biomarker for pancreatic adenocarcinoma
}

\author{
Xiuhui Shi ${ }^{1}$, Yan Zhao ${ }^{1}$, Ruizhi He ${ }^{1}$, Min Zhou ${ }^{1}$, Shutao Pan ${ }^{1}$, Shuo Yu ${ }^{1}$, Yu Xie ${ }^{1}, X$ \\ Li $^{1}$, Min Wang ${ }^{1}$, Xingjun Guo ${ }^{1}$ and Renyi Qin ${ }^{1}$ \\ ${ }^{1}$ Department of Biliary-Pancreatic Surgery, Affiliated Tongji Hospital, Tongji Medical College, Huazhong University of Science \\ and Technology, Wuhan, China \\ Correspondence to: Renyi Qin, email: ryqin@tjh.tjmu.edu.cn \\ Xingjun Guo, email: xjguo@tjh.tjmu.edu.cn
}

Keywords: long noncoding RNA; pancreatic adenocarcinoma; TCGA; ceRNA; prognosis

Received: August 23, 2017 Accepted: February 01, 2018 Epub: February 08, 2018 Published: May 11, 2018

Copyright: Shi et al. This is an open-access article distributed under the terms of the Creative Commons Attribution License 3.0 (CC BY 3.0), which permits unrestricted use, distribution, and reproduction in any medium, provided the original author and source are credited.

\section{ABSTRACT}

Pancreatic adenocarcinoma (PAAD) is a highly aggressive and metastatic cancer characterized by poor survival rates. Long non-coding RNAs (IncRNAs) play important roles in various biological processes, including cancer and PAAD. To identify the specific IncRNAs associated with PAAD and analyze their function, we constructed a global triple network based on the competitive endogenous RNA (ceRNA) theory and RNA-seq data from The Cancer Genome Atlas. Using 182 PAAD cases, we established a IncRNA-miRNA-mRNA co-expression network, which was composed of 43 IncRNA nodes, 253 mRNA nodes, 11 miRNA nodes, and 475 edges. Six IncRNAs in the ceRNA network were closely related to overall survival, and a three-IncRNA signature predicted survival of PAAD patients. Protein-protein interaction network data revealed that five genes were associated with overall survival. These results provide novel insight into the function of a IncRNA-associated ceRNA network in the pathogenesis of PAAD, and indicate that the identified three-IncRNA signature may serve as an independent prognostic marker in PAAD.

\section{INTRODUCTION}

Pancreatic adenocarcinoma (PAAD) and ductal adenocarcinoma, the most common and deadly forms of human pancreatic cancer, are highly invasive and metastatic, and are associated with poor survival [1]. Due to the aggressive nature of PAAD and development of chemoresistance, PAAD is the fourth leading cause of cancerrelated mortality in the western world, with an overall 5-year survival rate around $4 \%[2,3]$. Recent advances in genomic cancer research have led to numerous biomarker discoveries and improvements in patient care. However, in PAAD, specific biomarkers are still urgently needed.

Long noncoding RNAs (lncRNAs) are RNA transcripts that are longer than $200 \mathrm{nt}$ and exhibit limited or no protein-coding capacity. Many lncRNAs are uniquely expressed in differentiated tissues or specific cancer types [4-6]. LncRNAs drive many important cancer phenotypes by interacting with other cellular molecules, including DNA,
RNA, and proteins [7, 8]. Recent studies have demonstrated that IncRNAs are associated with the pathogenesis of different diseases, including PAAD [9-11]. Compared to protein-coding genes, lncRNAs have exhibited a superior potential as diagnostic and prognostic biomarkers. For example, IncRNAs have been used as diagnostic and prognostic biomarkers in hepatocellular carcinoma, gastric cancer, ovarian cancer, and prostate cancer [12-15].

Although thousands of IncRNAs have been discovered and recorded in public databases, such as NONCODE, LNCipedia, and LncRNADB, the functional characterization of lncRNAs is still in its infancy; up to now, only few lncRNAs have been functionally characterized $[16,17]$. It has been suggested that functionally related IncRNAs are associated with functionally related mRNAs or miRNAs, and involved in similar diseases. This association has been demonstrated in several diseases, but has not been studied in PAAD [18-20]. 
In this study, we have constructed a lncRNArelated network using the data from The Cancer Genome Atlas (TCGA), and the recently developed competitive endogenous RNA (ceRNA) theory [21].

\section{RESULTS}

\section{Identification of differentially expressed RNAs in PAAD}

A total of 182 samples were analyzed in this study, including 178 pancreatic adenocarcinoma tissues and 4 matched normal tissues. Using the cut-off criteria $(P<0.05$ and $\mid$ Fold change $\mid>2.0), 650$ differentially expressed lncRNAs (DELs), 50 differentially expressed miRNAs (DEMis), and 1724 differentially expressed mRNAs (DEMs) were identified between pancreatic adenocarcinoma tissues and matched normal tissues (Supplementary Tables 1-3). Altogether, 220 overexpressed and 430 under-expressed lncRNAs, 29 overexpressed and 21 under-expressed miRNAs, and 643 over-expressed and 1081 under-expressed mRNAs were identified. In order to verify the $P$-value and fold change using a different test, we used the volcano plot (Figure 1). Unsupervised hierarchic cluster analysis revealed that pancreatic adenocarcinoma tissues could be distinguished from normal tissues based on differentially expressed RNAs patterns (Figure 2).

\section{Construction of IncRNA-miRNA-mRNA network}

In order to establish lncRNA-miRNA-mRNA ceRNA network, lncRNAs and mRNAs targeted by miRNAs were identified from the above data. The flow chart for ceRNA network construction is shown in Figure 3. The results showed that 43 specific lncRNAs interacted with 13 specific
miRNAs (Supplementary Table 4). Target mRNAs of the 13 miRNAs were predicted using the TargetScan, miRDB, and miRTarBase databases. A total of 3715 target mRNAs of 13 miRNAs were identified Supplementary Table 5. Among the 3715 target mRNAs, 253 mRNAs were identified as DEMs. To elucidate the functions of the lncRNAs acting as miRNA targets, a network of lncRNAs, miRNAs, and mRNAs was constructed and visualized with Cytoscape. As shown in Figure 4, the lncRNA-miRNA-mRNA network was composed of 43 lncRNA nodes, 13 miRNA nodes, 253 mRNA nodes, and 475 edges.

\section{Identification of survival-related IncRNAs in PAAD}

To identify the lncRNAs associated with an overall survival (OS) in PAAD, we evaluated the association between lncRNAs expression and OS in 177 PAAD patients using Kaplan-Meier curve and Log-rank test. The results showed that four lncRNAs (HOPPIT, ABHD11AS1, MIR205HG, and LINC00460) negatively correlated with OS, and two miRNAs (MIR600HG and C9orf139) positively correlated with OS (Figure 5).

\section{Prognostic value of six IncRNAs risk score in PAAD}

We constructed a prognostic signature by integrating the expression profiles of 6 lncRNAs and corresponding estimated regression coefficients. Then, we calculated risk scores for each patient, and ranked them according to increased scores. Using multivariate Cox regression analysis, our results indicated that a three-lncRNA (LINC00460, C9orf139, and MIR600HG) signature might provide a powerful information for the prognosis of PAAD patients. Expression of the three prognostic lncRNAs is shown in the heat-map (Figure 6A). A total of 177 patients
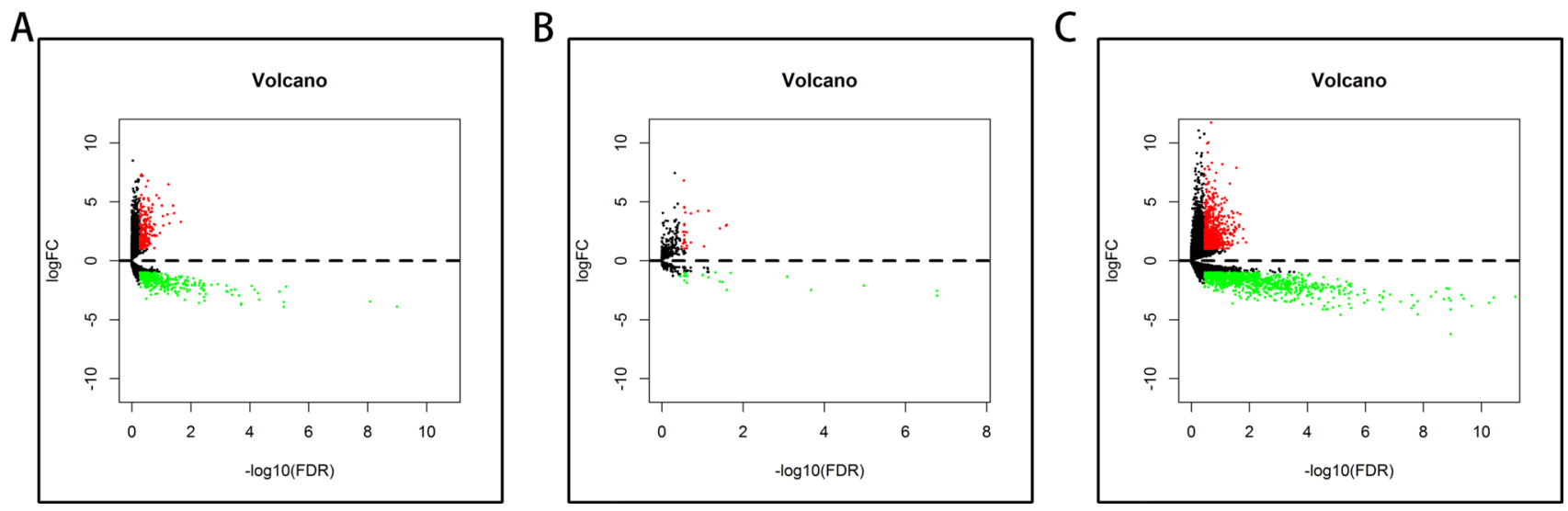

Figure 1: Volcano plot of the differentially expressed IncRNAs, miRNAs, and mRNAs between pancreatic adenocarcinoma and control tissues. Red dots indicate high expression and green dots indicate low expression of lncRNAs, miRNAs, and mRNAs. Black dots show the lncRNAs with expression of Fold change $<2$. The $\mathrm{X}$ axis represents an adjusted FDR and the $\mathrm{Y}$ axis represents the value of $\log 2$ FC. (A) Differentially expressed lncRNAs; (B) Differentially expressed miRNAs; (C) Differentially expressed mRNAs. 
A

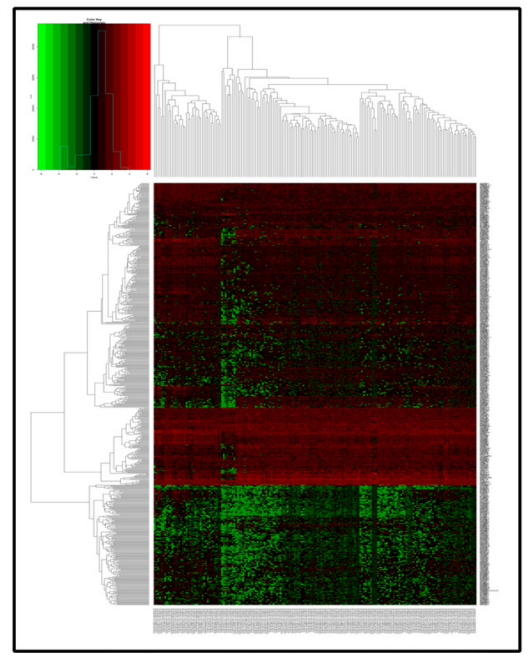

B

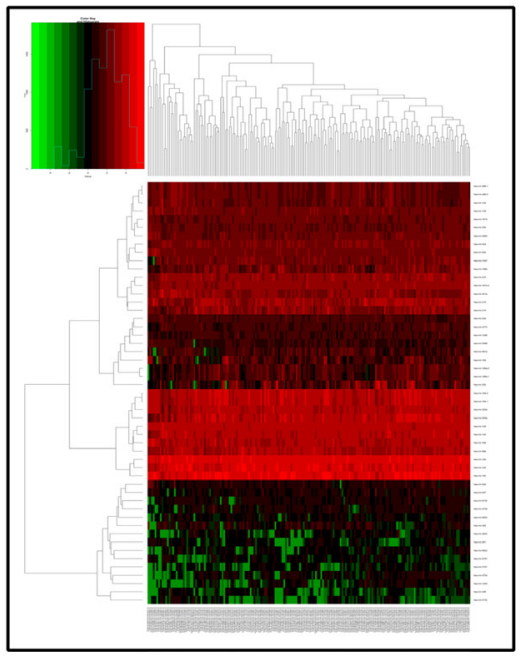

C

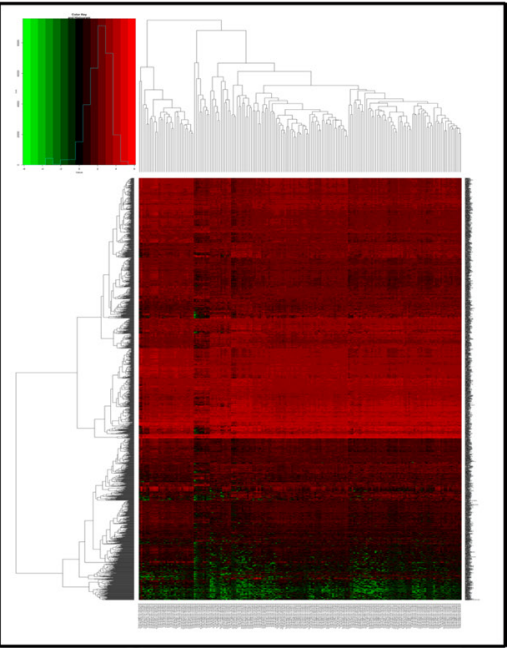

Figure 2: Hierarchical clustering of pancreatic adenocarcinoma and control tissues by differentially expressed RNAs. The heat-map consists of 4 normal tissues (left part) and 178 pancreatic adenocarcinoma tissues (right part). Each row represents an RNA level, and each column represents a sample. Red stands for high expression and green indicates low expression of lncRNAs, miRNAs, or mRNAs. (A) Differentially expressed lncRNAs; (B) Differentially expressed miRNAs; (C) Differentially expressed mRNAs.

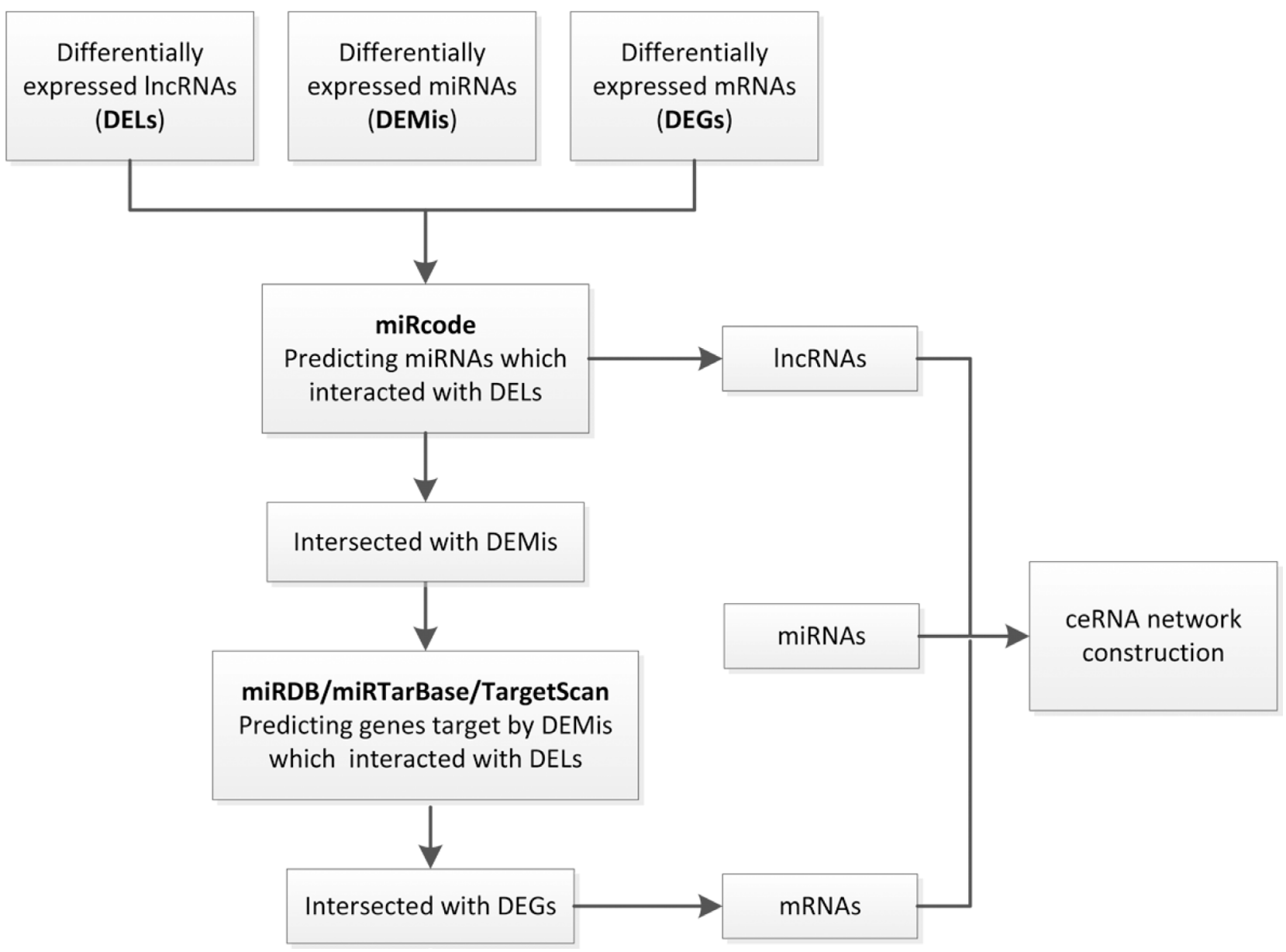

Figure 3: A flowchart of ceRNA network construction. (i) DELs, DEMis and DEMs with fold change $>2.0$ and $P$-value $<0.05$ were used; (ii) miRNA-IncRNA interactions were predicted by miRcode; (iii) lncRNAs not associated with DEMis were removed; (iv) mRNAs targeted by miRNAs were identified using miRDB, miRTarBase and TargetScan databases; (v) mRNAs that were not DEMs were removed; (vi) ceRNA network was constructed. 
with intact survival information were classified into a high risk group $(n=88)$ and a low risk group $(n=89)$ according to the median risk scores. Survival analysis was performed using the Kaplan-Meier method with a Log-rank statistical test. The results showed that patients in the high risk group had significantly worse OS than patients in the low risk group (Figure 6B). Receiver operating characteristic (ROC) curve was used to test the effect of the three-lncRNA signature (high risk vs. low risk) on OS (Figure 6C).

\section{Functional implication of prognostic IncRNA signature using enrichment analysis}

We performed enrichment analyses to elucidate the biological function of co-expressed DEMs of the lncRNAs in the network. Gene ontology (GO) analysis revealed that 70 enriched clusters were associated with biological processes (BP), 12 with cellular components (CC), and 20 with molecular function (MF). The top ten enriched clusters are shown in Figure 7A-7C. The first enriched biological process was humoral immune response. The first enriched cellular component and molecular function were integral component of membrane and protein kinase activity, respectively. Figure 7D displays the relationship between statistically significant top 30 DEMs and their related GO terms. In addition, a total of 42 KEGG pathways were enriched. The first enriched KEGG pathway was the Cytokinecytokine receptor interaction signaling pathway. The top ten enriched functional analyses are shown in Figure 8A and $8 \mathrm{~B}$. The top 4 functional enrichment analyses of the DEMs network are shown in Figure 8C.

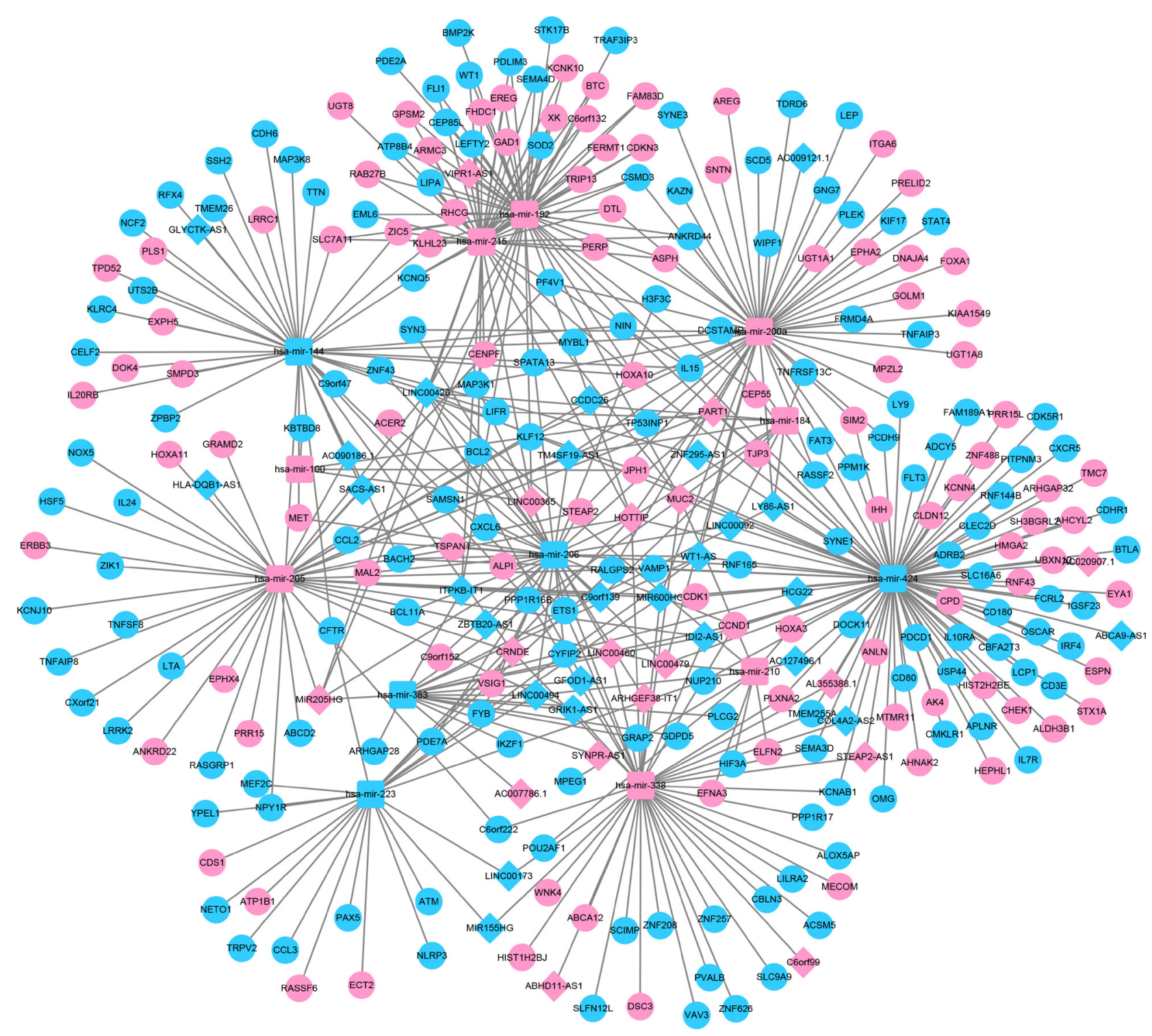

Figure 4: Illustration of the IncRNA-miRNA-mRNA network. The rhombus represents lncRNAs, the circle represents mRNAs, and the square represents miRNAs. There were 43 lncRNAs, 13 miRNAs, 253 mRNAs and 475 edges in the network. 


\section{Functional implication of prognostic IncRNA signature using PPI network construction}

STRING (a database of known and predicted protein interactions) was used to predict protein-protein interactions (PPI) among the DEMs. First, the 253 DEMs in the network were submitted to the STRING website. Then, the obtained PPI data with combined scores greater than 0.900 were selected for constructing PPI networks and disconnected nodes in the network were hidden. In the PPI networks, 8 node proteins, CDK1, ADCY5, GNG7, HIST1H2BJ, VAV3, CXCR5, GRAP2 and HIST2H2BE, showed a strong association with other node proteins (more than 5), indicating that they have higher hub degrees (Figure 9A, 9B). Among the 8 hub genes, 5 genes (CDK1, ADCY5, GNG7, HIST1H2BJ, and GRAP2) were associated with OS, indicating that they might play important roles in the PAAD.

\section{DISCUSSION}

Recent advances in genomic cancer research have led to numerous biomarker discoveries and improvements in patient care. For example, Oncotype DX, a 21-gene signature, has been used to predict estrogen receptor positive breast cancer recurrence in patients with lymph node negative disease; patients with high risk scores are recommended for adjuvant chemotherapy [22]. While there have been successful stories of biomarker application for clinical use in breast cancer [22], lung cancer [23] and melanoma [24], sadly, the clinical application of biomarkers in PAAD is very limited.

Aberrant expression of lncRNAs has been widely observed in various diseases, and dysregulated lncRNAs have emerged as key regulators of vital biological functions in cancer cells $[25,26]$. However, only a few studies have described lncRNA profiles in PAAD by RNAseq data analysis [27]. Song et al. [28] have constructed lncRNA-mRNA network by using dysregulated lncRNAs and mRNAs, and the ceRNA hypothesis. To date, only a few studies have investigated the interaction between IncRNAs and mRNAs, or lncRNAs and miRNAs in PAAD. Results from those studies have indicated that IncRNAs play a key role in competitive endogenous RNA (ceRNA) networks, but such ceRNA networks are still poorly characterized $[29,30]$. The ceRNA hypothesis has been proposed as a novel regulatory mechanism functioning through miRNA competition [31]. Recent
A

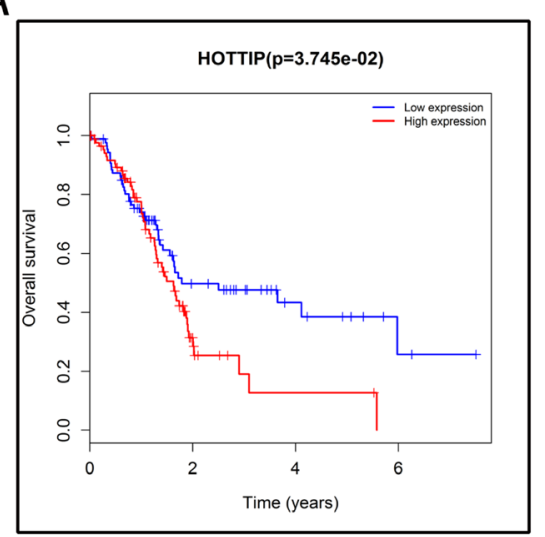

D

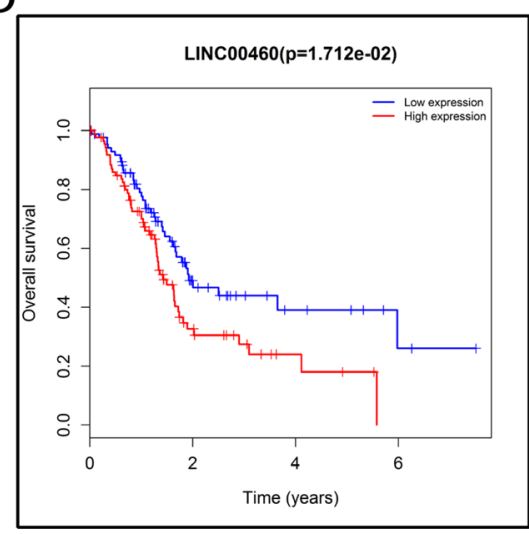

B

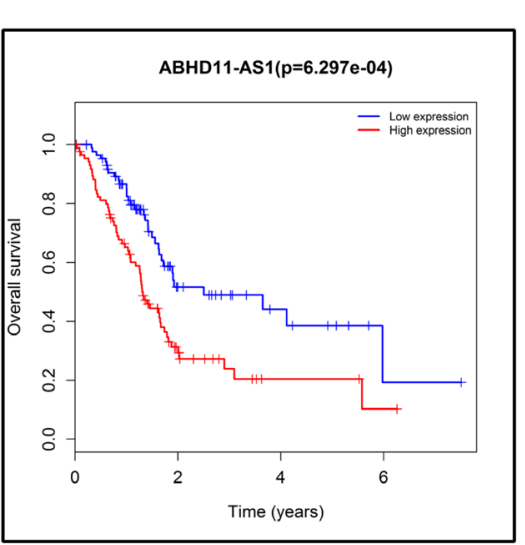

$\mathrm{E}$

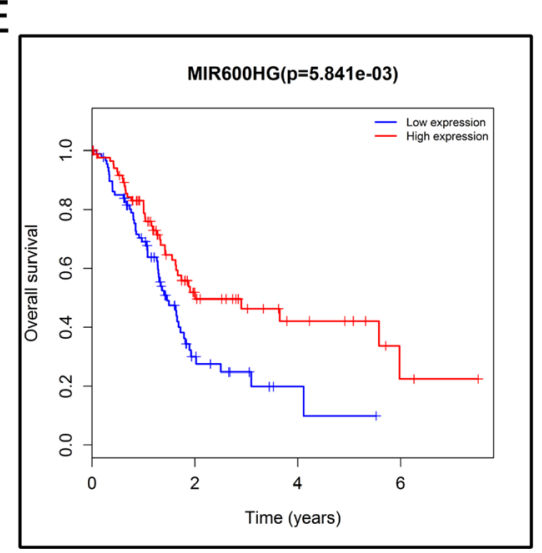

C

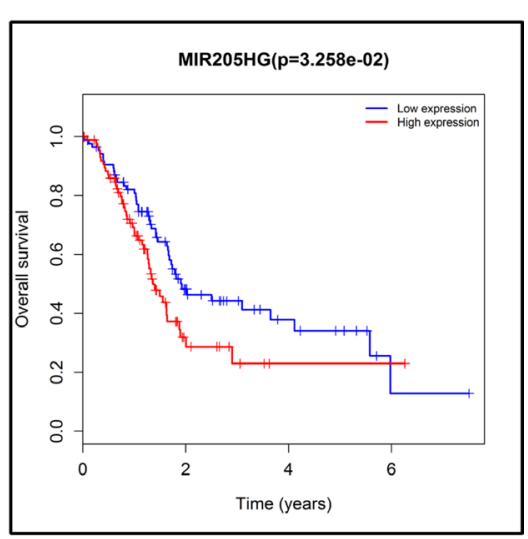

$\mathrm{F}$

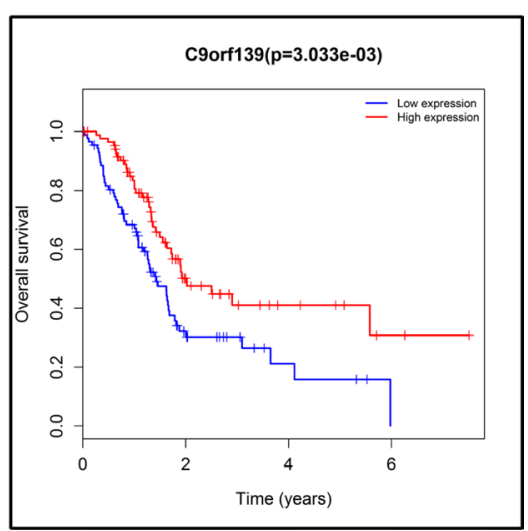

Figure 5: Six IncRNAs were associated with overall survival in 177 PAAD patients by using Kaplan-Meier curve and Log-rank test. The patients were stratified into high level group and low level group according to median of each lncRNA. (A) HOPPIT; (B) ABHD11-AS1; (C) MIR205HG; (D) LINC00460; (E) MIR600HG; (F) C9orf139. 
studies of ceRNA cross-talks have indicated that ceRNAs are regulated by miRNAs, which interact with lncRNAs in complex ceRNA networks [32].

In the present study, we identified lncRNAs, miRNAs and mRNAs differentially expressed in PAAD from TCGA database. Then, we constructed the PAADspecific lncRNA-miRNA-mRNA ceRNA network, which provides an important insight about the key RNAs of the ceRNA-mediated gene regulatory network in the initiation and development of PAAD. We analyzed OS of PAAD patients using 43 differentially expressed lncRNAs in the network; six of the lncRNAs were associated with OS in PAAD patients. After multivariate Cox regression analysis, the three-lncRNA (LINC00460, miR205HG and c3orf133) signature was established and identified as an independent prognostic factor for PAAD patients. ROC curve was used to test the effect of this three-lncRNA signature on OS.

Gene ontology (GO) analysis indicated that the aberrantly expressed genes mainly function in metabolism and immune response. Based on KEGG pathway analysis, several cancer-related pathways were detected, including p53 signaling pathway, JAK-STAT pathway, and T cell receptor signaling pathway. $\mathrm{Hu}$ et al. [33] reported that fenofibrate inhibited pancreatic cancer cell proliferation via accumulation of $\mathrm{p} 53$ protein and activation of $\mathrm{p} 53$ pathway mediated by upregulation of lncRNA MEG3. Denley et al. [34] found that activation of the IL-6R/Jak/Stat pathway was associated with a poor outcome in resected pancreatic ductal adenocarcinoma. In addition, it was reported that the $T$ cell receptor signaling pathway was associated with the PAAD pathogenesis $[35,36]$. Through PPI network construction, we found that several proteins formed the network, including CDK1, ADCY5, GNG7, HIST1H2BJ, VAV3, CXCR5, GRAP2 and HIST2H2BE; 5 of these hub genes (CDK1, ADCY5, GNG7, HIST1H2BJ and GRAP2) were associated with OS, indicating importance of this ceRNA network in PAAD.

Recent studies have indicated that lncRNAs function as crucial components of ceRNA networks by modulating other RNA transcripts [19, 37]. For example, HOTAIR may act as an endogenous sink by binding miR-331-3p, thereby abolishing the miRNAinhibitory activity and introducing an additional level of post-transcriptional regulation [38]. Hence, a potential connection between lncRNA, miRNA and mRNA may exist during the PAAD pathogenesis. In the present study,

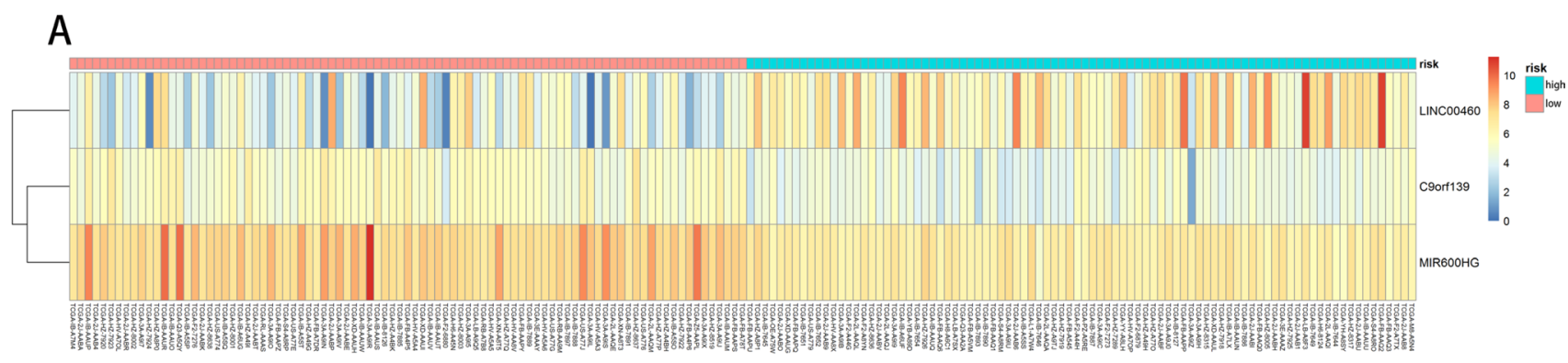

B

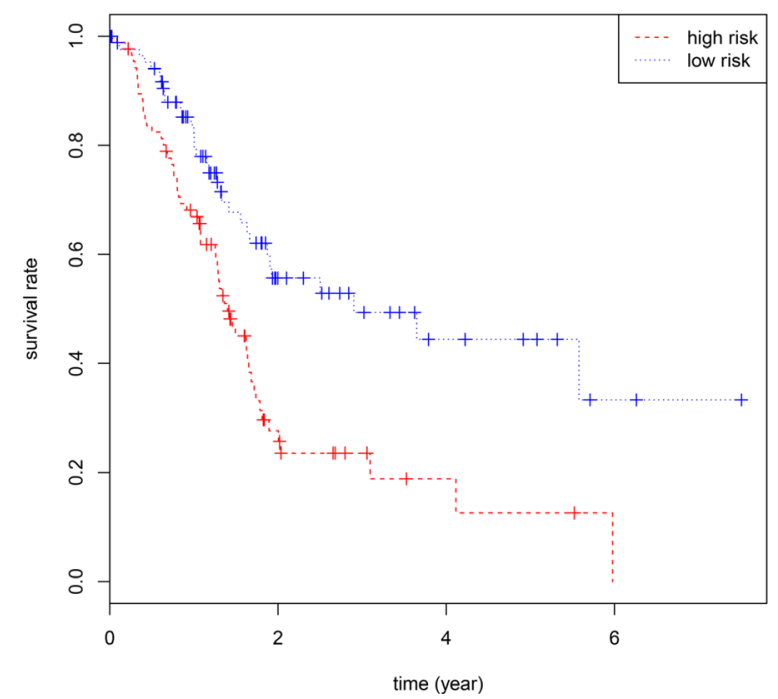

C

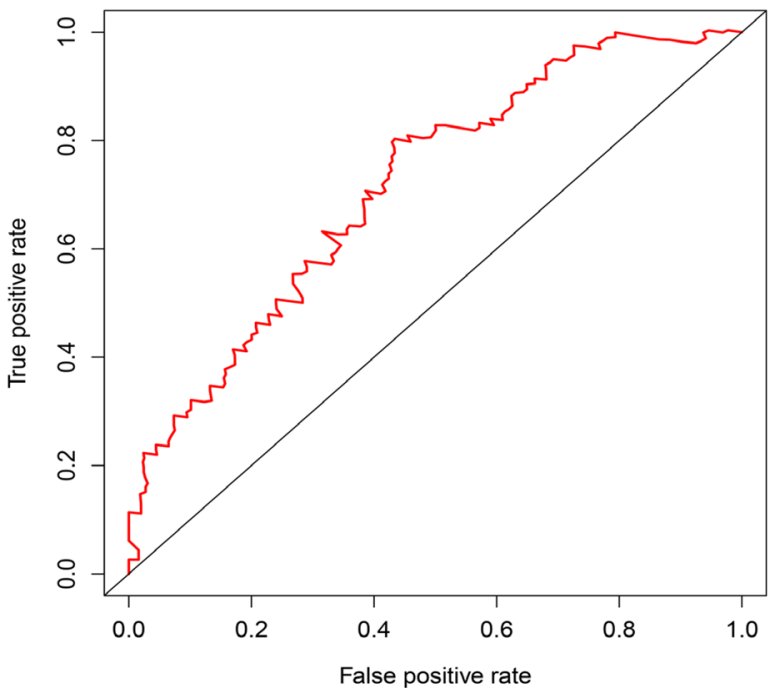

Figure 6: Prognostic evaluation of the 3-IncRNA signature in PAAD patients. (A) Expression heat-map of 3 prognostic IncRNAs. (B) Kaplan-Meier survival curve analysis for overall survival of PAAD patients using the 3-lncRNA signature. (C) ROC curve analysis of the 3-IncRNA signature. 

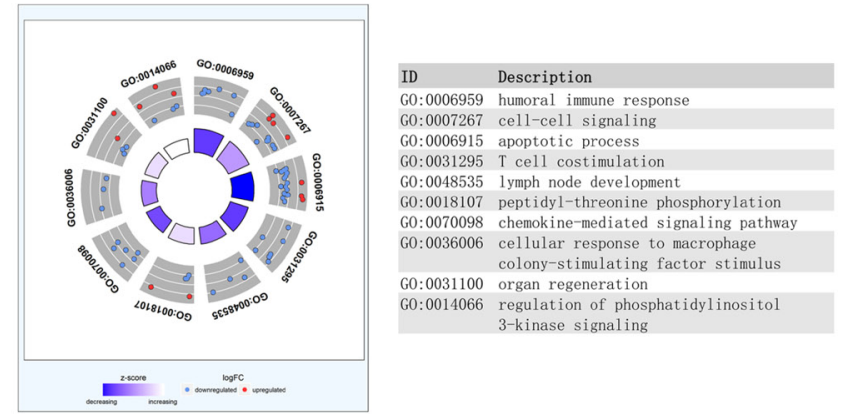

C

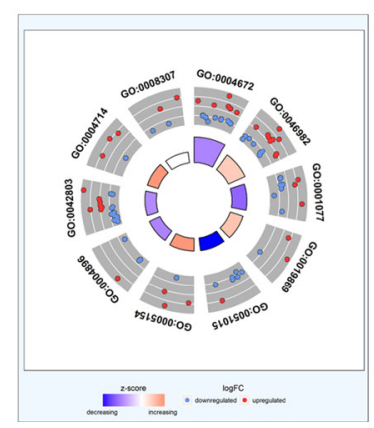

B
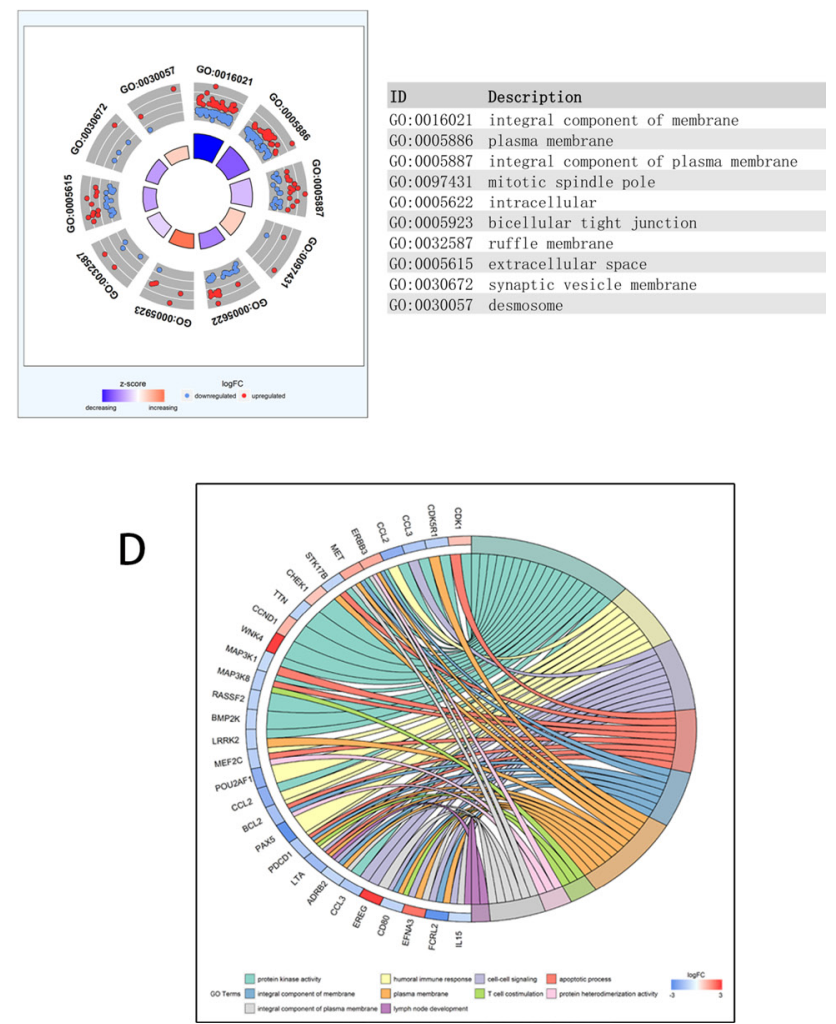

Figure 7: Functional analysis of key IncRNAs in PAAD. The outer circle shows a scatter plot of the expression (logFC) of differentially expressed mRNAs in each enriched gene ontology (GO) term: red circles indicate upregulation and blue circles indicate downregulation. The inner ring is a bar plot where the height of the bar indicates the significance of GO terms (log10-adjusted $P$ value), and color corresponds to the z-score: blue, decreased; red, increased; and white, unchanged.(A) Biological process (BP); (B) CC; (C) MF. (D) The plot shows the relationship between statistically significant top 30 mRNAs and their related GO terms; for each gene, the logFC value is shown by red/blue colored rectangles.

A

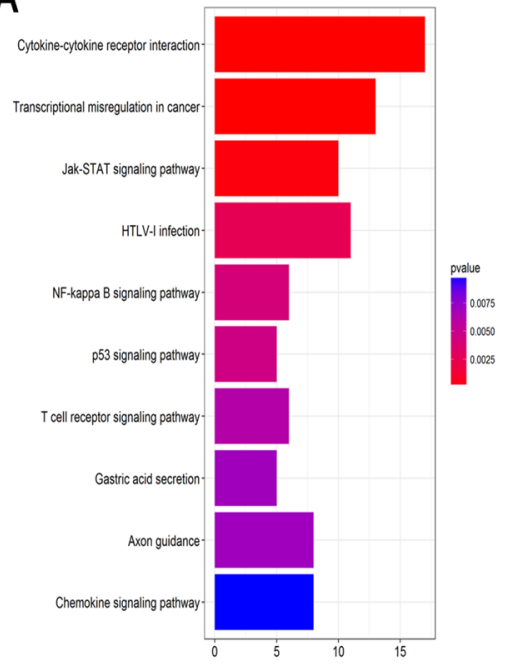

B

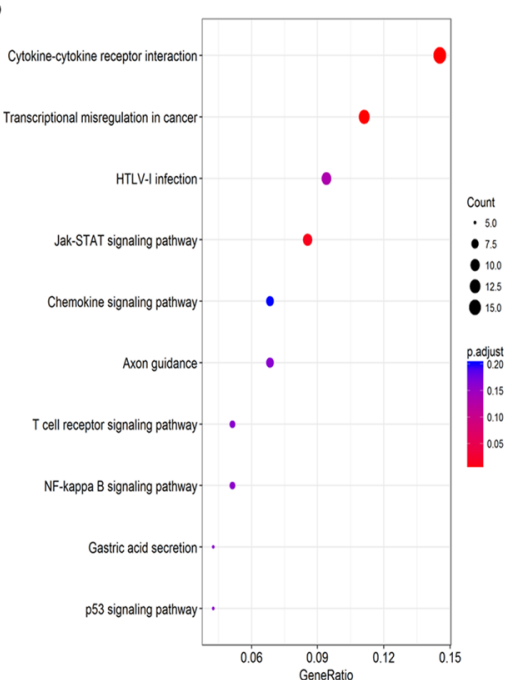

C

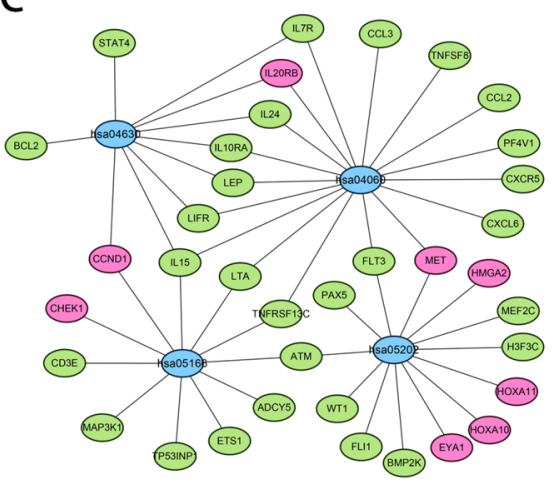

ID $\quad$ Description

hsa05202 Transcriptional misregulation in cancer

hsa04630 Jak-STAT signaling pathway

hsa05166 HTLV-I infection

Figure 8: KEGG pathway functional enrichment analysis of IncRNA-related DEMs in the network. (A, B) Top ten pathways of IncRNA-related DEMs. (C) Gene-concept networks by KEGG analysis of differentially expressed mRNAs, using Cytoscape. Blue hubs correspond to the most enriched KEGG pathway; red and green nodes represent upregulation and downregulation of mRNAs, respectively. 
we constructed the IncRNA-miRNA-mRNA ceRNA network to reveal a novel ceRNA regulatory network in PAAD. With respect to the associations between 43 cancer specific lncRNAs from ceRNA network and patient survival, we found that $6 \operatorname{lncRNAs}$ were related to OS, and three of them might serve as prognostic biomarkers for PAAD patients. Among these 3 lncRNAs, only LINC00460 has been reported in the regulatory networks in carcinoma; the other lncRNAs (MIR600HG and C3orf139) have not been reported [39]. Furthermore, a recent study has shown that LINC00460 is up-regulated in several cancers, including pancreatic cancer, breast cancer, lung cancer, colon cancer and bladder cancer [40], indicating that LINC00460 may play an important role in PAAD. Several cancer specific lncRNAs identified in the above ceRNA network, such as PART1 [41], LINC00356 [42], CCDC26 [43], and HOTTIP [44-46] have been identified as potential diagnostic and prognostic cancer biomarkers. In addition, WT-AS [47], LINC00092 [48], ABHD11-AS1 [49], and MIR205HG [50] have been associated with cancer initiation and progression.

In conclusion, we have searched for PAAD-specific lncRNAs by using a large scale analysis of hundreds of candidate lncRNAs in TCGA database, and identified aberrant expression profiles of cancer specific lncRNAs that correlated with OS. The identified PAAD-specific lncRNAs might serve as potential biomarkers in PAAD. Importantly, we have constructed the IncRNA-miRNA-mRNA ceRNA network to evaluate the ceRNA function in PAAD. We have found that three lncRNAs are differentially expressed in PAAD, and associated with an overall survival in PAAD patients. While efficacy of a single marker has been limited, multi-markers based models may provide a better prognostic information. Finally, we have constructed the three-lncRNA signature that correlates with PAAD patient
A

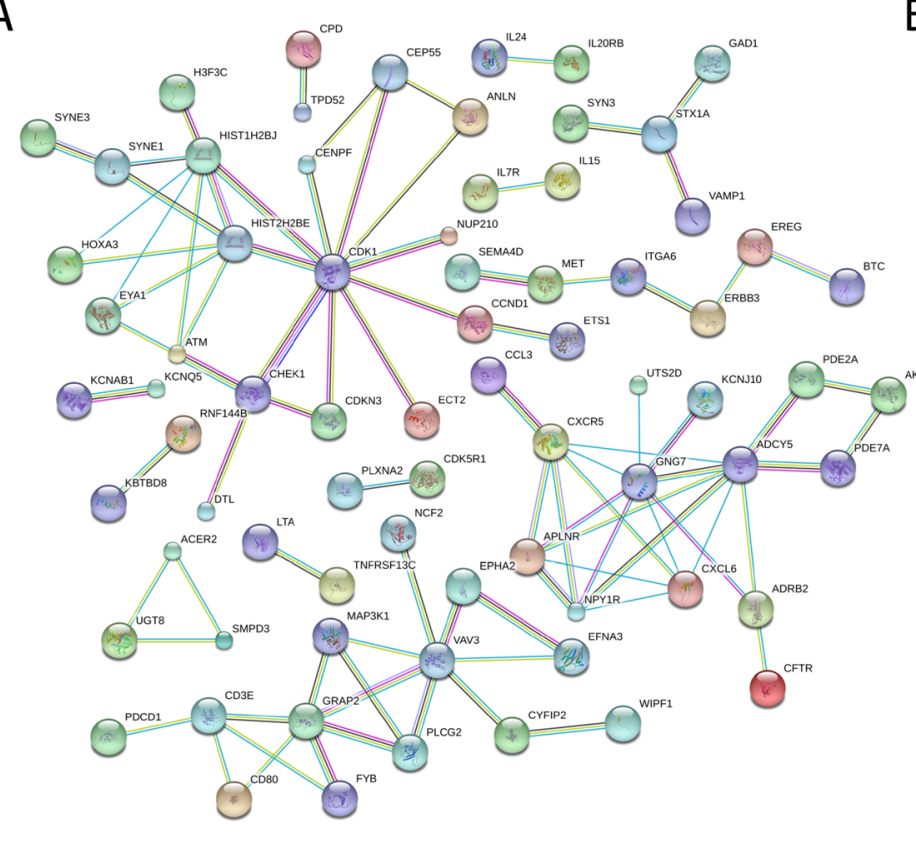

B

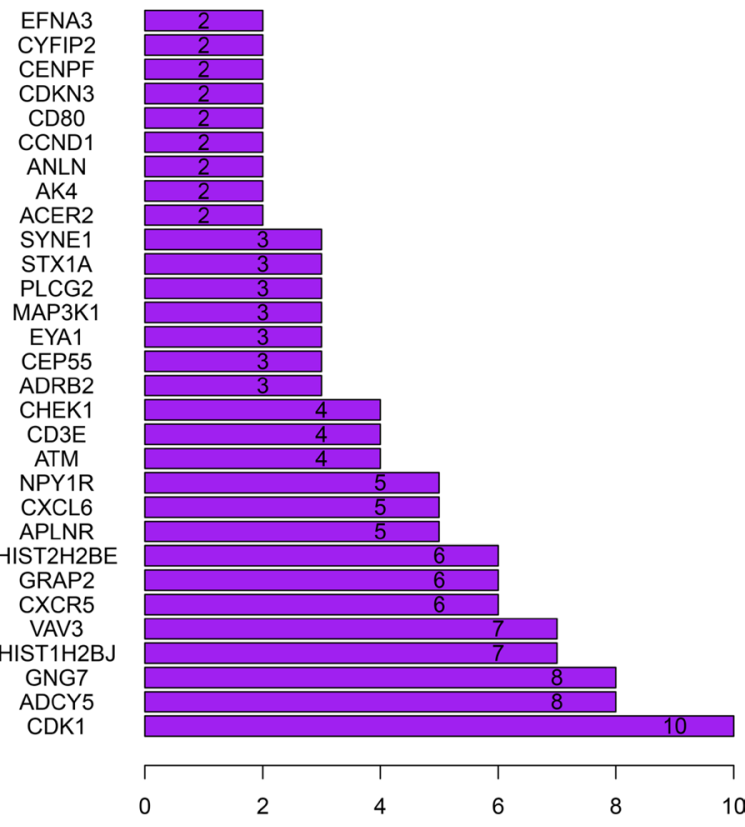

C

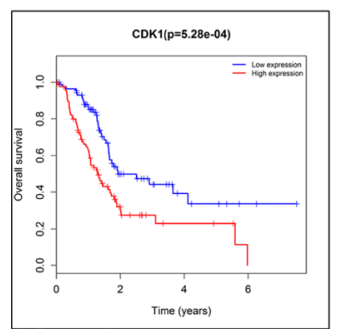

D

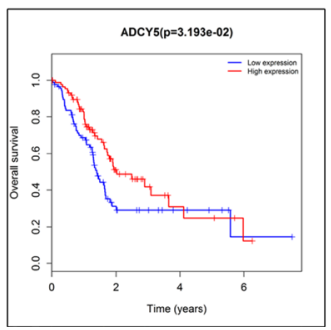

E

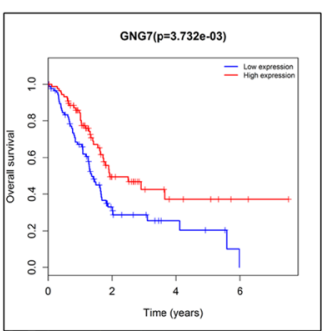

G

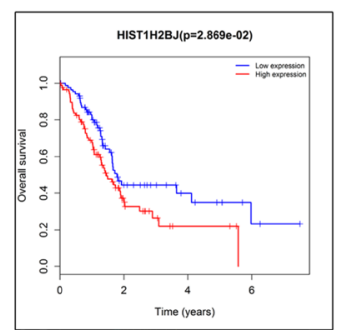

F

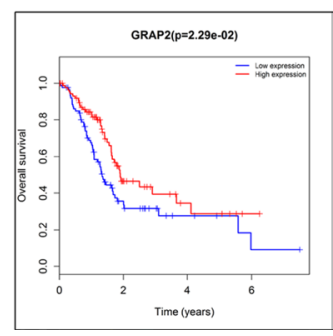

Figure 9: STRING database analysis of the PPI network of DEMs. (A, B) CDK1, ADCY5, GNG7, HIS1T1H2BJ, VAV3, CXCR5, GRAP2 and HIST2H2H2BE show increased association with other node proteins (more than 5), indicating that they have higher hub degrees. The patients were stratified into high level group and low level group according to median of each lncRNA. (C) CDK1; (D) ADCY5; (E) GNG7; (F) HIS1T1H2BJ; (G) GRAP2. 
survival, indicating that it may serve as an independent prognostic marker in PAAD.

\section{MATERIALS AND METHODS}

\section{RNA sequence data collection and processing}

The raw sequencing data and clinical information were downloaded from TCGA database (https://cancergenome. nih.gov/). The inclusion criteria were set as follows: (1) the sample included both miRNA sequencing data and clinical information; (2) the sample included prognosis information. A total of 182 samples were analyzed in this study, including 178 pancreatic adenocarcinoma tissues and 4 matched normal tissues. The RNA sequencing data were processed using $\mathrm{R}$ language package. The fold change (FC) values of individual RNA levels were calculated; differentially expressed RNAs with $|\mathrm{FC}|>2.0$ and $P$-value $<0.05$ were considered to be significant.

\section{Construction of IncRNA-miRNA-mRNA ceRNA network}

The ceRNA network was constructed based on the relationship between IncRNA, miRNA, and mRNA. To construct the ceRNA network, differentially expressed lncRNAs, miRNAs, and mRNAs with $|\mathrm{FC}|>2.0$ and $P$-value $<0.05$ were used. The miRcode database [51] was used to predict the lncRNA-miRNA interactions. The miRDB [52], Targetscan [53], and miRTarBase [54] databases were used to predict the mRNAs targeted by miRNAs. Furthermore, the predicted miRNAs and differentially expressed data of TCGA were combined to select the interacting lncRNAs and mRNAs. Cytoscape [55] was used to construct and visualize the lncRNAmiRNA-mRNA ceRNA network.

\section{Analysis of IncRNAs in the network and patient prognosis}

The differentially expressed lncRNA profiles were normalized by $\log 2$ transformation. The prognostic value of each differentially expressed lncRNA was evaluated using Kaplan-Meier curve and Log-rank method. The lncRNAs associated with overall survival (OS) were identified as candidates of prognostic lncRNA signature, and subjected to multivariate Cox regression analysis. Using the lncRNA signature, PAAD patients were classified into high risk and low risk groups using the median risk score. Differences in OS between the high risk and low risk groups were evaluated by Kaplan-Meier method. Receiver operating characteristic (ROC) curve was used to test the effect of the lncRNA signature (high risk vs. low risk) on OS. The area under the curve (AUC) under binomial exact confidence interval was calculated to generate the ROC curve.

\section{Functional enrichment analysis}

Using annotation, visualization, and integrated discovery (DAVID) database, the functional enrichment analyses of 253 differentially expressed mRNAs (DEMs) in the network, and gene ontology (GO) function analysis were carried out. Kyoto Encyclopedia of Genes and Genomes (KEGG) pathway enrichment analysis was carried out for those 253 DEMs in the network using the database of KOBAS [56]. In the GO analysis, the categories included biological process (BP), cellular component (CC), and molecular function (MF), and $P$-value $<0.05$ was regarded as statistically significant. To add quantitative molecular information to GO of interest, we used GOCircle and GOChord plot functions of GOplot R package [57], which permits to incorporate data derived from expression analysis with those obtained from the functional annotation enrichment analysis. In the KEGG pathways analysis, enriched pathways were identified according to the hypergeometric distribution with a $P$-value $<0.01$, and were analyzed using the cluster Profiler package [57]. In addition, to provide a readable graphic representation of the complex relationships between DEMs and relative KEGG pathway, the "pathway-gene network" was constructed by Cytoscape.

\section{Protein-protein interaction analysis by STRING}

Protein products of the 253 DEMs in the network were analyzed by the online tool, STRING [58], to predict protein-protein interactions. A combined score of not $<$ 0.9 (highest confidence score) was considered significant. The hub protein was selected based on its association with other proteins. DEMs associated with other DEMs indicated corresponding protein-protein interactions (PPI).

\section{Statistical analysis}

Statistical analysis was performed using the R 3.4.1 software. The data were expressed as mean \pm standard deviation (SD), and analyzed using the paired $t$-test. The significance level was set as 0.001 as a default to control the false discovery rate (FDR). $P$-value $<0.05$ was considered statistically significant.

\section{CONFLICTS OF INTEREST}

The authors declare no potential conflicts of interest.

\section{FUNDING}

This study was funded by The National Natural Science Foundation of China (Grants No. 81272659 to Renyin Qin, 81602475 to Xingjun Guo). 


\section{REFERENCES}

1. Smith K. Pancreatic cancer: FASCINating insights into the metastatic nature of pancreatic cancer. Nat Rev Gastroenterol Hepatol. 2014; 11:139. https://doi. org/10.1038/nrgastro.2014.15.

2. Jemal A, Siegel R, Xu J, Ward E. Cancer statistics, 2010. CA Cancer J Clin. 2010; 60:277-300. https://doi.org/10.3322/ caac. 20073.

3. Bardeesy N, DePinho RA. Pancreatic cancer biology and genetics. Nat Rev Cancer. 2002; 2:897-909. https:/doi. org/10.1038/nrc949.

4. Mercer TR, Dinger ME, Mattick JS. Long non-coding RNAs: insights into functions. Nat Rev Genet. 2009; 10:155-9. https://doi.org/10.1038/nrg2521.

5. Iyer MK, Niknafs YS, Malik R, Singhal U, Sahu A, Hosono Y, Barrette TR, Prensner JR, Evans JR, Zhao S, Poliakov A, Cao X, Dhanasekaran SM, et al. The landscape of long noncoding RNAs in the human transcriptome. Nat Genet. 2015; 47:199-208. https://doi.org/10.1038/ng.3192.

6. Ponting CP, Oliver PL, Reik W. Evolution and functions of long noncoding RNAs. Cell. 2009; 136:629-41. https://doi. org/10.1016/j.cell.2009.02.006

7. Wang KC, Chang HY. Molecular mechanisms of long noncoding RNAs. Mol Cell. 2011; 43:904-14. https://doi. org/10.1016/j.molcel.2011.08.018.

8. Schmitt AM, Chang HY. Long Noncoding RNAs in Cancer Pathways. Cancer Cell. 2016; 29:452-63. https://doi. org/10.1016/j.ccell.2016.03.010.

9. Sun YW, Chen YF, Li J, Huo YM, Liu DJ, Hua R, Zhang JF, Liu W, Yang JY, Fu XL, Yan T, Hong J, Cao H. A novel long non-coding RNA ENST00000480739 suppresses tumour cell invasion by regulating OS-9 and HIF-1alpha in pancreatic ductal adenocarcinoma. Br J Cancer. 2014; 111:2131-41. https://doi.org/10.1038/bjc.2014.520.

10. Ye Y, Chen J, Zhou Y, Fu Z, Zhou Q, Wang Y, Gao W, Zheng S, Zhao X, Chen T, Chen R. High expression of AFAP1-AS1 is associated with poor survival and shortterm recurrence in pancreatic ductal adenocarcinoma. J Transl Med. 2015; 13:137. https://doi.org/10.1186/ s12967-015-0490-4.

11. Li CH, Xiao Z, Tong JH, To KF, Fang X, Cheng AS, Chen Y. EZH2 coupled with HOTAIR to silence MicroRNA-34a by the induction of heterochromatin formation in human pancreatic ductal adenocarcinoma. Int J Cancer. 2017; 140:120-9. https://doi.org/10.1002/ijc.30414.

12. Gu Y, Chen T, Li G, Yu X, Lu Y, Wang H, Teng L. LncRNAs: emerging biomarkers in gastric cancer. Future Oncol. 2015; 11:2427-41. https://doi.org/10.2217/fon.15.175.

13. Li J, Wang X, Tang J, Jiang R, Zhang W, Ji J, Sun B. HULC and Linc00152 Act as Novel Biomarkers in Predicting Diagnosis of Hepatocellular Carcinoma. Cell Physiol Biochem. 2015; 37:687-96. https://doi.org/10.1159/000430387.
14. Guo Q, Cheng Y, Liang T, He Y, Ren C, Sun L, Zhang G. Comprehensive analysis of lncRNA-mRNA co-expression patterns identifies immune-associated lncRNA biomarkers in ovarian cancer malignant progression. Sci Rep. 2015; 5:17683. https://doi.org/10.1038/srep17683.

15. Cheng WS, Tao H, Hu EP, Liu S, Cai HR, Tao XL, Zhang L, Mao JJ, Yan DL. Both genes and lncRNAs can be used as biomarkers of prostate cancer by using high throughput sequencing data. Eur Rev Med Pharmacol Sci. 2014; 18:3504-10.

16. Koirala P, Huang J, Ho TT, Wu F, Ding X, Mo YY. LncRNA AK023948 is a positive regulator of AKT. Nat Commun. 2017; 8:14422. https://doi.org/10.1038/ncomms14422.

17. Kawasaki Y, Komiya M, Matsumura K, Negishi L, Suda S, Okuno M, Yokota N, Osada T, Nagashima T, Hiyoshi M, Okada-Hatakeyama M, Kitayama J, Shirahige K, Akiyama T. MYU, a Target lncRNA for Wnt/c-Myc Signaling, Mediates Induction of CDK6 to Promote Cell Cycle Progression. Cell Reports. 2016; 16:2554-64.

18. Hua Y, Ma X, Liu X, Yuan X, Qin H, Zhang X. Identification of the potential biomarkers for the metastasis of rectal adenocarcinoma. APMIS. 2017; 125:93-100. https://doi.org/10.1111/apm.12633.

19. Li CY, Liang GY, Yao WZ, Sui J, Shen X, Zhang YQ, Peng H, Hong WW, Ye YC, Zhang ZY, Zhang WH, Yin LH, $\mathrm{Pu}$ YP. Integrated analysis of long non-coding RNA competing interactions reveals the potential role in progression of human gastric cancer. Int J Oncol. 2016; 48:1965-76. https://doi.org/10.3892/ijo.2016.3407.

20. Liu D, Yu X, Wang S, Dai E, Jiang L, Wang J, Yang Q, Yang F, Zhou S, Jiang W. The gain and loss of long noncoding RNA associated-competing endogenous RNAs in prostate cancer. Oncotarget. 2016; 7:57228-38. https:// doi.org/10.18632/oncotarget.11128.

21. Ma MZ, Chu BF, Zhang Y, Weng MZ, Qin YY, Gong W, Quan ZW. Long non-coding RNA CCAT1 promotes gallbladder cancer development via negative modulation of miRNA-218-5p. Cell Death Dis. 2015; 6:e1583. https://doi. org/10.1038/cddis.2014.541.

22. Paik S, Tang G, Shak S, Kim C, Baker J, Kim W, Cronin M, Baehner FL, Watson D, Bryant J, Costantino JP, Geyer CE Jr, Wickerham DL, et al. Gene expression and benefit of chemotherapy in women with node-negative, estrogen receptor-positive breast cancer. J Clin Oncol. 2006; 24:3726-34. https://doi.org/10.1200/jco.2005.04.7985.

23. Langer CJ. Epidermal growth factor receptor inhibition in mutation-positive non-small-cell lung cancer: is afatinib better or simply newer? J Clin Oncol. 2013; 31:3303-6. https://doi.org/10.1200/jco.2013.49.8782.

24. Chapman PB, Hauschild A, Robert C, Haanen JB, Ascierto P, Larkin J, Dummer R, Garbe C, Testori A, Maio M, Hogg D, Lorigan P, Lebbe C, et al, and BRIM-3 Study Group. 
Improved survival with vemurafenib in melanoma with BRAF V600E mutation. N Engl J Med. 2011; 364:2507-16. https://doi.org/10.1056/NEJMoa1103782.

25. Harries LW. Long non-coding RNAs and human disease. Biochem Soc Trans. 2012; 40:902-6. https://doi. org/10.1042/bst20120020.

26. Esteller M. Non-coding RNAs in human disease. Nat Rev Genet. 2011; 12:861-74. https://doi.org/10.1038/nrg3074.

27. Yao K, Wang Q, Jia J, Zhao H. A competing endogenous RNA network identifies novel mRNA, miRNA and lncRNA markers for the prognosis of diabetic pancreatic cancer. Tumour Biol. 2017; 39:1010428317707882. https://doi. org/10.1177/1010428317707882.

28. Song C, Zhang J, Liu Y, Pan H, Qi HP, Cao YG, Zhao JM, Li S, Guo J, Sun HL, Li CQ. Construction and analysis of cardiac hypertrophy-associated lncRNA-mRNA network based on competitive endogenous RNA reveal functional IncRNAs in cardiac hypertrophy. Oncotarget. 2016; 7:10827-40. https://doi.org/10.18632/oncotarget.7312.

29. Zhou M, Diao Z, Yue X, Chen Y, Zhao H, Cheng L, Sun J. Construction and analysis of dysregulated lncRNAassociated ceRNA network identified novel lncRNA biomarkers for early diagnosis of human pancreatic cancer. Oncotarget. 2016; 7:56383-94. https://doi.org/10.18632/ oncotarget.10891.

30. Fu Z, Li G, Li Z, Wang Y, Zhao Y, Zheng S, Ye H, Luo Y, Zhao X, Wei L, Liu Y, Lin Q, Zhou Q, Chen R. Endogenous miRNA Sponge LincRNA-ROR promotes proliferation, invasion and stem cell-like phenotype of pancreatic cancer cells. Cell Death Dis. 2017; 3:17004.

31. Tay Y, Rinn J, Pandolfi PP. The multilayered complexity of ceRNA crosstalk and competition. Nature. 2014; 505:344-52. https://doi.org/10.1038/nature12986.

32. Karreth FA, Pandolfi PP. ceRNA cross-talk in cancer: when ce-bling rivalries go awry. Cancer Discov. 2013; 3:1113-21. https://doi.org/10.1158/2159-8290.cd-13-0202.

33. Hu D, Su C, Jiang M, Shen Y, Shi A, Zhao F, Chen R, Shen Z, Bao J, Tang W. Fenofibrate inhibited pancreatic cancer cells proliferation via activation of p53 mediated by upregulation of LncRNA MEG3. Biochem Biophys Res Commun. 2016; 471:290-5. https://doi.org/10.1016/j. bbrc.2016.01.169.

34. Denley SM, Jamieson NB, McCall P, Oien KA, Morton JP, Carter CR, Edwards J, McKay CJ. Activation of the IL-6R/ $\mathrm{Jak} /$ stat pathway is associated with a poor outcome in resected pancreatic ductal adenocarcinoma. J Gastrointest Surg. 2013; 17:887-98. https://doi.org/10.1007/ s11605-013-2168-7.

35. Schmielau J, Nalesnik MA, Finn OJ. Suppressed T-cell receptor zeta chain expression and cytokine production in pancreatic cancer patients. Clin Cancer Res. 2001; $7: 933 \mathrm{~s}-9 \mathrm{~s}$.
36. Mohammed S, Sukumaran S, Bajgain P, Watanabe N, Heslop HE, Rooney CM, Brenner MK, Fisher WE, Leen AM, Vera JF. Improving Chimeric Antigen Receptor-Modified T Cell Function by Reversing the Immunosuppressive Tumor Microenvironment of Pancreatic Cancer. Mol Ther. 2017; 25:249-58. https://doi.org/10.1016/j.ymthe.2016.10.016.

37. Ergun S, Oztuzcu S. Oncocers: ceRNA-mediated cross-talk by sponging miRNAs in oncogenic pathways. Tumour Biol. 2015; 36:3129-36. https://doi.org/10.1007/s13277-015-3346-X.

38. Liu XH, Sun M, Nie FQ, Ge YB, Zhang EB, Yin DD, Kong R, Xia R, Lu KH, Li JH, De W, Wang KM, Wang ZX. Lnc RNA HOTAIR functions as a competing endogenous RNA to regulate HER2 expression by sponging miR-331-3p in gastric cancer. Mol Cancer. 2014; 13:92. https://doi. org/10.1186/1476-4598-13-92.

39. Zhang Y, Tao Y, Liao Q. Long noncoding RNA: a crosslink in biological regulatory network. Brief Bioinform. 2017 Apr 24 [Epub ahead of print]. https://doi.org/10.1093/bib/ bbx042.

40. Cao W, Liu JN, Liu Z, Wang X, Han ZG, Ji T, Chen WT, Zou X. A three-lncRNA signature derived from the Atlas of ncRNA in cancer (TANRIC) database predicts the survival of patients with head and neck squamous cell carcinoma. Oral Oncol. 2017; 65:94-101. https://doi.org/10.1016/j. oraloncology.2016.12.017.

41. Zhang XQ, Sun S, Lam KF, Kiang KM, Pu JK, Ho AS, Lui WM, Fung CF, Wong TS, Leung GK. A long noncoding RNA signature in glioblastoma multiforme predicts survival. Neurobiol Dis. 2013; 58:123-31. https://doi. org/10.1016/j.nbd.2013.05.011.

42. Li Y, He Y, Han S, Liang Y. Identification and Functional Inference for Tumor-associated Long Non-coding RNA. IEEE/ACM Trans Comput Biol Bioinform. 2017 Mar 24 [Epub ahead of print]. https://doi.org/10.1109/ TCBB.2017.2687442.

43. Peng W, Jiang A. Long noncoding RNA CCDC26 as a potential predictor biomarker contributes to tumorigenesis in pancreatic cancer. Biomed Pharmacother. 2016; 83:712-7. https://doi.org/10.1016/j.biopha.2016.06.059.

44. Ye H, Liu K, Qian K. Overexpression of long noncoding RNA HOTTIP promotes tumor invasion and predicts poor prognosis in gastric cancer. Onco Targets Ther. 2016; 9:2081-8. https://doi.org/10.2147/ott.s95414.

45. Quagliata L, Matter MS, Piscuoglio S, Arabi L, Ruiz C, Procino A, Kovac M, Moretti F, Makowska Z, Boldanova T, Andersen JB, Hammerle M, Tornillo L, et al. Long noncoding RNA HOTTIP/HOXA13 expression is associated with disease progression and predicts outcome in hepatocellular carcinoma patients. Hepatology. 2014; 59:911-23. https://doi.org/10.1002/hep.26740.

46. Ren YK, Xiao Y, Wan XB, Zhao YZ, Li J, Li Y, Han GS, Chen XB, Zou QY, Wang GC, Lu CM, Xu YC, Wang YC. Association of long non-coding RNA HOTTIP with 
progression and prognosis in colorectal cancer. Int J Clin Exp Pathol. 2015; 8:11458-63.

47. Du T, Zhang B, Zhang S, Jiang X, Zheng P, Li J, Yan M, Zhu Z, Liu B. Decreased expression of long non-coding RNA WT1-AS promotes cell proliferation and invasion in gastric cancer. Biochim Biophys Acta. 2016; 1862:12-9. https://doi.org/10.1016/j.bbadis.2015.10.001.

48. Zhao L, Ji G, Le X, Wang C, Xu L, Feng M, Zhang Y, Yang H, Xuan Y, Yang Y, Lei L, Yang Q, Lau WB, et al. Long Noncoding RNA LINC00092 Acts in CancerAssociated Fibroblasts to Drive Glycolysis and Progression of Ovarian Cancer. Cancer Res. 2017; 77:1369-82. https:// doi.org/10.1158/0008-5472.can-16-1615.

49. Chen M, Li J, Zhuang C, Cai Z. Increased lncRNA ABHD11-AS1 represses the malignant phenotypes of bladder cancer. Oncotarget. 2017; 8:28176-86. https://doi. org/10.18632/oncotarget.14945.

50. Zou F, Li J, Jie X, Peng X, Fan R, Wang M, Wang J, Liu Z, Li H, Deng H, Yang X, Luo D. Rs3842530 Polymorphism in MicroRNA-205 Host Gene in Lung and Breast Cancer Patients. Med Sci Monit. 2016; 22:4555-5464. https://doi. org/10.12659/msm.901042.

51. Jeggari A, Marks DS, Larsson E. miRcode: a map of putative microRNA target sites in the long non-coding transcriptome. Bioinformatics. 2012; 28:2062-3. https:// doi.org/10.1093/bioinformatics/bts344.

52. Wong N, Wang X. miRDB: an online resource for microRNA target prediction and functional annotations. Nucleic Acids Res. 2015; 43:D146-52. https://doi. org/10.1093/nar/gku1104.
53. Agarwal V, Bell GW, Nam JW, Bartel DP. Predicting effective microRNA target sites in mammalian mRNAs. eLife. 2015; 4. https://doi.org/10.7554/eLife.05005.

54. Chou CH, Chang NW, Shrestha S, Hsu SD, Lin YL, Lee WH, Yang CD, Hong HC, Wei TY, Tu SJ, Tsai TR, Ho SY, Jian TY, et al. miRTarBase 2016: updates to the experimentally validated miRNA-target interactions database. Nucleic Acids Res. 2016; 44:D239-47. https:// doi.org/10.1093/nar/gkv1258.

55. Shannon P, Markiel A, Ozier O, Baliga NS, Wang JT, Ramage D, Amin N, Schwikowski B, Ideker T. Cytoscape: a software environment for integrated models of biomolecular interaction networks. Genome Res. 2003; 13:2498-504. https://doi.org/10.1101/gr.1239303.

56. Xie C, Mao X, Huang J, Ding Y, Wu J, Dong S, Kong L, Gao G, Li CY, Wei L. KOBAS 2.0: a web server for annotation and identification of enriched pathways and diseases. Nucleic Acids Res. 2011; 39:W316-22. https:// doi.org/10.1093/nar/gkr483.

57. Walter W, Sanchez-Cabo F, Ricote M. GOplot: an R package for visually combining expression data with functional analysis. Bioinformatics. 2015; 31:2912-4. https://doi.org/10.1093/bioinformatics/btv300.

58. Szklarczyk D, Morris JH, Cook H, Kuhn M, Wyder S, Simonovic M, Santos A, Doncheva NT, Roth A, Bork P, Jensen LJ, von Mering C. The STRING database in 2017: quality-controlled protein-protein association networks, made broadly accessible. Nucleic Acids Res. 2017; 45:D362-D8. https://doi.org/10.1093/nar/gkw937. 